\title{
Pseudo-Hermitian Matrix Exactly Solvable Hamiltonian
}

\author{
Ancilla Nininahazwe \\ Institut de Pédagogie Appliquée, Université du Burundi, Bujumbura, Burundi \\ Email: nininaha@yahoo.fr
}

How to cite this paper: Nininahazwe, A. (2019) Pseudo-Hermitian Matrix Exactly Solvable Hamiltonian. Open Journal of Microphysics, 9, 1-9.

https://doi.org/10.4236/ojm.2019.91001

Received: January 9, 2019

Accepted: January 28, 2019

Published: January 31, 2019

Copyright (c) 2019 by author(s) and Scientific Research Publishing Inc. This work is licensed under the Creative Commons Attribution International License (CC BY 4.0).

http://creativecommons.org/licenses/by/4.0/

\begin{abstract}
The non $P T$-symmetric exactly solvable Hamiltonian describing a system of a fermion in the external magnetic field which couples to a harmonic oscillator through some pseudo-hermitian interaction is considered. We point out all properties of both of the original Mandal and the original Jaynes-Cummings Hamitonians. It is shown that these Hamiltonians are respectively pseudo-hermitian and hermitian [1] [2]. Like the direct approach to invariant vector spaces used in Refs. [3] [4], we reveal the exact solvability of both the Mandal and Jaynes-Cummings Hamiltonians after expressing them in the position operator and the impulsion operator.
\end{abstract}

\section{Keywords}

Pseudo-Hermiticity, Exact Solvability, Direct Method

\section{Introduction}

Several new theoretical aspects in quantum mechanics have been developed in last years. In the series of papers [5] [6], it is shown that the traditional self adjointness requirement (i.e. the hermiticity property) of a Hamilton operator is not necessary condition to guarantee real eigenvalues and that the weaker condition PT-symmetry of the Hamiltonian is sufficient for the purpose. Following the theory developed in Refs. [5] [6], let's remind that a Hamiltonian is invariant under the action of the combined parity operator $P$ and the time reversal operator $T$ if the relation $H^{P T}=H$ is proved (i.e. $P T$-symmetry is said to be broken). As a consequence, the spectrum associated the previous Hamiltonian is entirely real.

An alternative property called pseudo-hermiticity for a Hamiltonian to be associated to a real spectrum is shown in details in the Refs. [1] [2]. 
Referring the ideas of [1] [2], we recall here that a Hamiltonian is said to be $\eta$ pseudo-hermitian if it satisfies the relation $\eta H \eta^{-1}=H^{+}$, where $\eta$ denotes an invertible linear hermitian operator.

Another direction of quantum mechanics is the notions of quasi exact solvability and exact solvability [7] [8] [9] [10].

In the last few years, a new class of operators has been discovered. This class is intermediate between exactly solvable operators and non solvable operators. Its name is the quasi-exactly solvable ( $Q E S$ ) operators, for which a finite part of the spectrum can be computed algebraically.

This paper is organized as follows:

In Section 2, we briefly describe the general model which is expressed in terms of the creation and the annihilation operators. We show that the Hamiltonian describing the model is pseudo-hermitian if $\phi=-1$, or it is hermitian if $\phi=+1$

In Section 3, we show in details the properties of the Mandal Hamiltonian namely the non-hermiticity, the non $P T$-symmetry, the pseudo-hermiticity and the exact solvability.

In Section 4, as in the previous section, it was pointed out that the original Jaynes-Cummings Hamiltonian is hermitian and exactly solvable.

\section{The Model}

In this section, we consider a Hamiltonian describing a system of a fermion in the external magnetic field, $\boldsymbol{B}$ which couples the harmonic oscillator interaction (i.e. $\hbar \omega a^{+} a$ ) and the pseudo-hermitian interaction if $\phi=-1$, or the hermitian interaction if $\phi=+1$ (i.e. $\left.\rho\left(\sigma_{+} a+\phi \sigma_{-} a^{+}\right)\right)$[1] [2]:

$$
H=\mu \boldsymbol{\sigma} \cdot \boldsymbol{B}+\hbar \omega a^{+} a+\rho\left(\sigma_{+} a+\phi \sigma_{-} a^{+}\right),
$$

where

$\sigma, \sigma_{ \pm}$denote Pauli matrices,

$\rho, \mu$ are real parameters,

$a^{+}$, a refer the creation and annihilation operators respectively satisfying the usual bosonic commutation relation

$$
\left[a, a^{+}\right]=1,[a, a]=\left[a^{+}, a^{+}\right]=0 \text { and } \sigma_{ \pm} \equiv \frac{1}{2}\left(\sigma_{x} \pm i \sigma_{y}\right)
$$

Recall that the matrices $\sigma_{+}, \sigma_{-}, \sigma_{x}, \sigma_{y}$ and $\sigma_{z}$ can be expressed in the following matrix forms:

$$
\sigma_{+}=\left(\begin{array}{ll}
0 & 1 \\
0 & 0
\end{array}\right), \sigma_{-}=\left(\begin{array}{ll}
0 & 0 \\
1 & 0
\end{array}\right), \sigma_{x}=\left(\begin{array}{ll}
0 & 1 \\
1 & 0
\end{array}\right), \sigma_{y}=\left(\begin{array}{cc}
0 & -i \\
i & 0
\end{array}\right), \sigma_{z}=\left(\begin{array}{cc}
1 & 0 \\
0 & -1
\end{array}\right)
$$

For the sake simplicity, one can choose the external field in the $z$-direction (i.e. $\left.\boldsymbol{B}=B_{0} \boldsymbol{z}\right)$ in order to reduce the Hamiltonian given by the Equation (1) and it becomes [1] [2]:

$$
H=\frac{\varepsilon}{2} \sigma_{z}+\omega a^{+} a+\rho\left(\sigma_{+} a+\phi \sigma_{-} a^{+}\right)
$$


with $\varepsilon=2 \mu B_{0}$ and $\hbar=1$.

\section{Properties of the Original Mandal Hamiltonian}

\subsection{The Non-Hermiticity}

In this section, we reveal that the Hamiltonian described by the Equation (3) is non- hermitian if $\phi=-1$. It is called Mandal Hamiltonian (i.e. $H_{M}$ ) and it takes the following form:

$$
H_{M}=\frac{\varepsilon}{2} \sigma_{z}+\omega a^{+} a+\rho\left(\sigma_{+} a-\sigma_{-} a^{+}\right)
$$

Taking account to the following identities:

$$
\begin{aligned}
& \left(a^{+}\right)^{+}=a \\
& (a)^{+}=a^{+}, \\
& \left(\sigma_{+}\right)^{+}=\sigma_{-}, \\
& \left(\sigma_{-}\right)^{+}=\sigma_{+},
\end{aligned}
$$

let's show that the Mandal Hamiltonian given by the above Equation (4) is non hermitian:

$$
\begin{gathered}
H_{M}^{+}=\left(\frac{\varepsilon}{2} \sigma_{z}\right)^{+}+\left(\omega a^{+} a\right)^{+}+\left[\rho\left(\sigma_{+} a-\sigma_{-} a^{+}\right)\right]^{+}, \\
H_{M}^{+}=\frac{\varepsilon}{2} \sigma_{z}+\omega a^{+} a-\rho\left(\sigma_{+} a-\sigma_{-} a^{+}\right) .
\end{gathered}
$$

Comparing the expressions given by the Equations (4) and (6), we see that they are different (i.e. $H_{M}^{+} \neq H_{M}$ ), as a consequence, we are allowed to conclude that the Mandal Hamiltonian $H_{M}$ is non-hermitian.

\subsection{The Non PT-Symmetry of $H_{M}$}

In this section, we prove that the Mandal Hamiltonian is non $P T$-symmetric [5] [6]. Recall that the parity operator is represented by the symbol $P$ and the time-reversal operator is described by the symbol $T$.

The effect of the parity operator $P$ implies the following changes [1] [2]:

$$
\begin{aligned}
& P \varepsilon P^{-1}=\varepsilon, P \sigma_{z} P^{-1}=\sigma_{z}, P \sigma_{+} P^{-1}=\sigma_{+}, \\
& P \sigma_{-} P^{-1}=\sigma_{-}, P a P^{-1}=-a, P a^{+} P^{-1}=-a^{+} .
\end{aligned}
$$

Notice also the changes of the following quantities under the effect of the time reversal operator $T$ :

$$
\begin{aligned}
& T \varepsilon T^{-1}=\varepsilon, T \sigma_{z} T^{-1}=-\sigma_{z}, T \sigma_{+} T^{-1}=-\sigma_{-}, \\
& T \sigma_{-} T^{-1}=-\sigma_{+}, T a T^{-1}=-a, T a^{+} T^{-1}=-a^{+} .
\end{aligned}
$$

Taking account to the relations (7) and (8), one can easily deduce the changes of the Mandal Hamiltonian under the effect of combined operators $P$ et $T$ as follows

$$
(P T) H_{M}(P T)^{-1}=(P T)\left[\frac{\varepsilon}{2} \sigma_{z}+\omega a^{+} a+\rho\left(\sigma_{+} a^{+}-\sigma_{-} a\right)\right](P T)^{-1},
$$




$$
(P T) H_{M}(P T)^{-1}=-\frac{\varepsilon}{2} \sigma_{z}+\omega a^{+} a+\rho\left(\sigma_{+} a^{+}-\sigma_{-} a\right)
$$

This above relation (9) can be written as follows

$$
H_{M}^{P T}=-\frac{\varepsilon}{2} \sigma_{z}+\omega a^{+} a+\rho\left(\sigma_{+} a^{+}-\sigma_{-} a\right)
$$

Comparing the relations (4) and (10), we see that they are different (i.e. $\left.H_{M}^{P T} \neq H_{M}\right)$, it means that the Mandal Hamiltonian $H_{M}$ is not invariant under the combined action of the parity operator $P$ and the time-reversal operator $T$. In other words, the Mandal Hamiltonian $H_{M}$ is not $P T$-symmetric.

\subsection{Pseudo-Hermiticity of $\boldsymbol{H}_{M}$}

In this section, we first prove that the non $P T$-symmetric Mandal Hamiltonian is pseudo-hermitian with respect to third Pauli matrix $\sigma_{z}$ [1] [2]:

$$
\begin{aligned}
\sigma_{z} H_{M} \sigma_{z}^{-1} & =\frac{\varepsilon}{2} \sigma_{z} \sigma_{z} \sigma_{z}^{-1}+\omega \sigma_{z} a^{+} a \sigma_{z}^{-1}+\rho\left(\sigma_{z} \sigma_{+} \sigma_{z}^{-1} a-\sigma_{z} \sigma_{-} \sigma_{z}^{-1} a^{+}\right) \\
& =\frac{\varepsilon}{2} \sigma_{z}+\omega a^{+} a-\rho\left(\sigma_{+} a-\sigma_{-} a^{+}\right)
\end{aligned}
$$

with $\sigma_{z} \sigma_{ \pm} \sigma_{z}^{-1}=-\sigma_{\mp}$ and $\sigma_{z}^{-1}=\sigma_{z}^{t}=\sigma_{z}$.

Comparing the Equations (6) and (11), it is seen that the following relation is satisfied:

$$
\sigma_{z} H_{M} \sigma_{z}^{-1}=H_{M}^{+}
$$

Taking account to this above relation, we are allowed to conclude that the Mandal Hamiltonian is pseudo-hermitian with respect to $\sigma_{z}$.

Finally, we reveal a pseudo-hermiticity of $H_{M}$ with respect to the parity operator $P$ :

$$
\begin{aligned}
P H_{M} P^{-1} & =\frac{\varepsilon}{2} P \sigma_{z} P^{-1}+\omega P a^{+} a P^{-1}+\rho\left(P \sigma_{+} a P^{-1}-P \sigma_{-} a^{+} P^{-1}\right) \\
& =\frac{\varepsilon}{2} \sigma_{z}+\omega a^{+} a-\rho\left(\sigma_{+} a-\sigma_{-} a^{+}\right) \\
& =H_{M}^{+}
\end{aligned}
$$

Here we have used the relations (7) in order to obtain this above equation (13). As a consequence, one can conclude that the Mandal Hamiltonian is pseudo-hermitian with respect to the parity operator $P$.

Note that even if $H_{M}$ is non hermitian and non $P T$-symmetric, its eigenvalues are entirely real due to the pseudo-hermiticity property [1].

\subsection{Differential Form and Exact Solvability of $\boldsymbol{H}_{M}$}

In this step, our purpose is to change the Mandal Hamiltonian given by the Equation (4) in appropriate differential operator (i.e. $H_{M}$ is expressed in the position operator $x$ and in the impulsion operator $\left.p=-i \frac{\mathrm{d}}{\mathrm{d} x}\right)$. Thus, referring to the ideas of exactly and quasi-exactly solvable operators studied in the Refs. [7] [8] 
[9] [10], we reveal that $H_{M}$ preserves a family of vector spaces of polynomials in the variable $x$.

With this aim, we use the usual representation of the creation and annihilation operators of the harmonic oscillator respectively $a^{+}$and $a$ [1] [2]:

$$
a^{+}=\frac{p+i m \omega x}{\sqrt{2 m \omega \hbar}}, \quad a=\frac{p-i m \omega x}{\sqrt{2 m \omega \hbar}}
$$

where $\omega$ is the oscillation frequency, $m$ denotes the mass, $x$ refers to the position operator and the impulsion operator is $p=-i \frac{\mathrm{d}}{\mathrm{d} x}, p^{2}=-\frac{\mathrm{d}^{2}}{\mathrm{~d} x^{2}}$.

Using appropriate units, we can assume $m=\hbar=1$ and the operators $a^{+}$ and $a$ take the following forms:

$$
a^{+}=\frac{p+i \omega x}{\sqrt{2 \omega}}, \quad a=\frac{p-i \omega x}{\sqrt{2 \omega}} .
$$

Replacing the operators $a^{+}$and $a$ by their expressions given by this above Equation (15) in the Equation (4), the Mandal Hamiltonian $H_{M}$ takes the following form:

$$
H_{M}=\frac{\varepsilon}{2} \sigma_{z}+\frac{p^{2}-\omega+\omega^{2} x^{2}}{2}+\rho \frac{\left[\sigma_{+}(p-i \omega x)-\sigma_{-}(p+i \omega x)\right]}{\sqrt{2 \omega}} .
$$

In order to reveal the exact solvability of the above operator $H_{M}$, we first perform the standard gauge transformation [2]:

$$
\tilde{H}_{M}=R^{-1} H_{M} R, \quad R=\exp \left(-\frac{\omega x^{2}}{2}\right) .
$$

After some algebraic manipulations, the new Hamiltonian $\tilde{H}_{M}$ (known as gauge Hamiltonian) is obtained

$$
\begin{aligned}
\tilde{H}_{M} & =\frac{\varepsilon}{2} \sigma_{z}-\frac{1}{2} \frac{\mathrm{d}^{2}}{\mathrm{~d} x^{2}}+\omega x \frac{\mathrm{d}}{\mathrm{d} x}+\rho \frac{\left[\sigma_{+} p-\sigma_{-}(p+2 i \omega x)\right]}{\sqrt{2 \omega}} \\
& =\frac{\varepsilon}{2} \sigma_{z}+\frac{p^{2}}{2}+i \omega x p+\rho \frac{\left[\sigma_{+} p-\sigma_{-}(p+2 i \omega x)\right]}{\sqrt{2 \omega}}
\end{aligned}
$$

Replacing the Pauli matrices $\sigma_{z}, \sigma_{+}$and $\sigma_{-}$by their respective expressions given by the relation (2), the final form of the gauge Hamiltonian is:

$$
\begin{gathered}
\tilde{H}_{M}=\frac{\varepsilon}{2}\left(\begin{array}{cc}
1 & 0 \\
0 & -1
\end{array}\right)+\left(\begin{array}{cc}
\frac{p^{2}}{2}+i \omega x p & 0 \\
0 & \frac{p^{2}}{2}+i \omega x p
\end{array}\right)-\rho\left(\begin{array}{cc}
0 & \frac{p}{\sqrt{2 \omega}} \\
\frac{p+2 i \omega x}{\sqrt{2 \omega}} & 0
\end{array}\right), \\
\tilde{H}_{M}=\left(\begin{array}{cc}
\frac{p^{2}}{2}+i \omega x p+\frac{\varepsilon}{2} & \rho \frac{p}{\sqrt{2 \omega}} \\
-\rho \frac{p+2 i \omega x}{\sqrt{2 \omega}} & \frac{p^{2}}{2}+i \omega x p-\frac{\varepsilon}{2}
\end{array}\right) .
\end{gathered}
$$

Note that one can easily check if this above gauge Hamiltonian $\tilde{H}_{M}$ preserves the vector spaces of polynomials $V_{n}=\left(P_{n-1}(x), P_{n}(x)\right)^{t}$ with $n \in \mathrm{N}$. As 
the integer $n$ doesn't have to be fixed (i.e. it is arbitrary), $\tilde{H}_{M}$ is exactly solvable. Indeed, its all eigenvalues can be computed algebraically. Even if the gauge Mandal Hamiltonian $\tilde{H}_{M}$ is non-hermitian and non $P T$-symmetric, its spectrum energy is entirely real due to the property of the pseudo-hermiticity [1] [2].

Thus, the vector spaces preserved by the operator $H_{M}$ have the following form

$$
W_{n}=\mathrm{e}^{-\frac{\omega x^{2}}{2}}\left(P_{n-1}(x), P_{n}(x)\right)^{t}
$$

where $P_{n-1}(x)$ and $P_{n}(x)$ denote respectively the polynomials of degree $n-1$ and $n$.

As the gauge Mandal Hamiltonian $\tilde{H}_{M}$, it is obvious that the original Mandal Hamiltonian $H_{M}$ is exactly solvable. Due to this property of exact solvability, the whole spectrum of $H_{M}$ can be computed exactly (i.e. by the algebraic methods) [1] [2] [3].

\section{Properties of the Jaynes-Cummings Hamiltonian}

\subsection{The Hermiticity}

In this section, considering $\phi=+1$, the Hamiltonian given by the Equation (3) leads to the standard Jaynes-Cummings Hamiltonian of the following form

$$
H_{J C}=\frac{\varepsilon}{2} \sigma_{z}+\omega a^{+} a+\rho\left(\sigma_{+} a+\sigma_{-} a^{+}\right)
$$

Our aim is now to prove that the above Hamiltonian $H_{J C}$ is hermitian.

Indeed, in order to reveal the hermiticity of the Jaynes-Cummings Hamiltonian given by the above relation (21), the following relation $H_{J C}^{+}=H_{J C}$ must be satisfied.

Consider now the following relation

$$
H_{J C}^{+}=\left(\frac{\varepsilon}{2} \sigma_{z}\right)^{+}+\left(\omega a^{+} a\right)^{+}+\left[\rho\left(\sigma_{+} a+\sigma_{-} a^{+}\right)\right]^{+},
$$

Taking account to the identities of the relation (5), this above equation leads the following expression:

$$
H_{J C}^{+}=\frac{\varepsilon}{2} \sigma_{z}+\omega a^{+} a+\left[\rho\left(\sigma_{+} a+\sigma_{-} a^{+}\right)\right] .
$$

Comparing the Equations (21) and (23), one can write that

$$
H_{J C}^{+}=H_{J C} \text {. }
$$

Referring to this equation (24), it is obvious that the standard Jaynes-Cummings Hamiltonian is hermitian. As a consequence, its eigenvalues are real due to the property of hermiticity.

\subsection{Differential Form and Exact Solvability of $\boldsymbol{H}_{J C}$}

Along the same lines as in the above section 3.4, our purpose is to change the Jaynes-Cummings Hamiltonian given by the Equation (21) in appropriate diffe- 
rential operator (i.e. $H_{J C}$ is expressed in the position operator $x$ and in the impulsion operator $\left.p=-i \frac{\mathrm{d}}{\mathrm{d} x}\right)$.

With this purpose, we use the usual expressions of the creation and annihilation operators of the harmonic oscillator respectively $a^{+}$and $a$ given by the Equation (15).

Substituting (15) in the Equation (21), the Jaynes-Cummings Hamiltonian $H_{J C}$ is written now as follows

$$
H_{J C}=\frac{\varepsilon}{2} \sigma_{z}+\frac{p^{2}-\omega+\omega^{2} x^{2}}{2}+\rho \frac{\left[\sigma_{+}(p-i \omega x)+\sigma_{-}(p+i \omega x)\right]}{\sqrt{2 \omega}}
$$

Operating on the above operator $H_{J C}$ the standard gauge transformation as

$$
\tilde{H}_{J C}=R^{-1} H_{J C} R, \quad R=\exp \left(-\frac{\omega x^{2}}{2}\right),
$$

after some algebraic manipulations, the new Hamiltonian $\tilde{H}_{J C}$ (known as gauge Hamiltonian) is obtained

$$
\begin{aligned}
\tilde{H}_{M} & =\frac{\varepsilon}{2} \sigma_{z}-\frac{1}{2} \frac{\mathrm{d}^{2}}{\mathrm{~d} x^{2}}+\omega x \frac{\mathrm{d}}{\mathrm{d} x}+\rho \frac{\left[\sigma_{+} p+\sigma_{-}(p+2 i \omega x)\right]}{\sqrt{2 \omega}} \\
& =\frac{\varepsilon}{2} \sigma_{z}+\frac{p^{2}}{2}+i \omega x p+\rho \frac{\left[\sigma_{+} p+\sigma_{-}(p+2 i \omega x)\right]}{\sqrt{2 \omega}}
\end{aligned}
$$

Replacing the Pauli matrices $\sigma_{z}, \sigma_{+}$and $\sigma_{-}$respectively by their matrix form given by the relation (2), the final form of the gauge Hamiltonian $\tilde{H}_{J C}$ is

$$
\begin{gathered}
\tilde{H}_{M}=\frac{\varepsilon}{2}\left(\begin{array}{cc}
1 & 0 \\
0 & -1
\end{array}\right)+\left(\begin{array}{cc}
\frac{p^{2}}{2}+i \omega x p & 0 \\
0 & \frac{p^{2}}{2}+i \omega x p
\end{array}\right)+\rho\left(\begin{array}{cc}
0 & \frac{p}{\sqrt{2 \omega}} \\
\frac{p+2 i \omega x}{\sqrt{2 \omega}} & 0
\end{array}\right), \\
\tilde{H}_{M}=\left(\begin{array}{cc}
\frac{p^{2}}{2}+i \omega x p+\frac{\varepsilon}{2} & \rho \frac{p}{\sqrt{2 \omega}} \\
\rho \frac{p+2 i \omega x}{\sqrt{2 \omega}} & \frac{p^{2}}{2}+i \omega x p-\frac{\varepsilon}{2}
\end{array}\right) .
\end{gathered}
$$

Note that one can easily check if this above gauge Hamiltonian $\tilde{H}_{J C}$ preserves the finite dimensional vector spaces of polynomials namely $V_{n}=\left(P_{n-1}(x), P_{n}(x)\right)^{t}$ with $n \in \mathrm{N}$. As the integer $n$ is arbitrary, the gauge Jaynes-Cummings Hamiltonian $\tilde{H}_{J C}$ is exactly solvable.

As a consequence, its all eigenvalues can be computed algebraically. Indeed, the vector spaces preserved by the operator $H_{J C}$ have the following form

$$
W_{n}=\mathrm{e}^{-\frac{\omega x^{2}}{2}}\left(P_{n-1}(x), P_{n}(x)\right)^{t}
$$

where $P_{n-1}(x)$ and $P_{n}(x)$ denote respectively the polynomials of degree $n-1$ and $n$.

As the gauge Jaynes-Cummings Hamiltonian $\tilde{H}_{J C}$, it is obvious that the 
standard Jaynes-Cummings Hamiltonian $H_{J C}$ is exactly solvable. In other words, all eigenvalues associated to the Hamiltonian $H_{J C}$ can be calculated algebraically (i.e. by the algebraic methods) [1-3].

\section{Conclusion}

In this paper, we have put out all properties of the original Mandal Hamiltonian. We have shown that the Mandal Hamiltonian $H_{M}$ is non-hermitian and non-invariant under the combined action of the parity operator $P$ and the time-reversal operator $T$. Even if the previous properties are not satisfied, it has been proved that the Mandal Hamiltonian $H_{M}$ is pseudo-hermitian with respect to $P$ and with respect to $\sigma_{3}$ also. With the direct method, we have revealed that $H_{M}$ preserves the finite dimensional vector spaces of polynomials namely $V_{n}=\left(P_{n-1}(x), P_{n}(x)\right)^{t}$. Indeed, the Mandal Hamiltonian $H_{M}$ is said to be exactly solvable [1] [2] [3] [4]. Along the same lines used in Section 3, we have pointed out that the standard Jaynes-Cummings Hamiltonian $H_{J C}$ is hermitian and exactly solvable in Section 4.

\section{Acknowledgements}

I thank Pr. Yves Brihaye of useful discussions.

\section{Conflicts of Interest}

The author declares no conflicts of interest regarding the publication of this paper.

\section{References}

[1] Mandal, B.P. (2005) Pseudo-Hermitian Interaction between an Oscillator and a Spin $-\frac{1}{2}$ Particle in the External Magnetic Field. Modern Physics Letters A, 20, 655-662. https://doi.org/10.1142/S0217732305016488

[2] Brihaye, Y. and Nininahazwe, A. (2006) Extended Jaynes-Cummings Models and Quasi-Exact Solvability. Journal of Physics A: Mathematical and General, 39, 1-14. https://doi.org/10.1088/0305-4470/39/31/011

[3] Rühl, W. and Turbiner, A. (1995) Exact Solvability of the Calogero and the Sutherland Models. Modern Physics Letters A, 10, 2213. https://doi.org/10.1142/S0217732395002374

[4] Gomez-Ullate, D., Kamran, N. and Milson, R. (2005) Quasi-Exact Solvability and the Direct Approach to Invariant Subspaces. Journal of Physics, A38, 2005-2019. https://doi.org/10.1088/0305-4470/38/9/011

[5] Bender, C. and Boettcher, S. (1998) Real Spectra in Non-Hermitian Hamiltonians having PT-Symmetry. Physical Reviews Letters, 80, 5243. https://doi.org/10.1103/PhysRevLett.80.5243

[6] Brihaye, Y. and Nininahazwe, A. (2004) On PT-Symmetric Extensions of the Calogero and the Sutherland Models. International Journal of Morden Physics, A19, 4391-4400. https://doi.org/10.1142/S0217751X04019858

[7] Turbiner, A.V. (1988) Quasi-Exactly Solvable Problems and sl(2) Algebra. Commu- 
nications in Mathematical Physics, 118, 467-474.

https://doi.org/10.1007/BF01466727

[8] Ushveridze, A.G. (1995) Quasi-Exactly Solvable Models in Quantum Mechanics. Institute of Physics Publishing.

[9] Brihaye, Y. and Kosinski, P. (1997) Quasi-Exactly Solvable Matrix Models in sl(n). Physics Letters B, 424, 43-47. https://doi.org/10.1016/S0370-2693(98)00167-1

[10] Brihaye, Y. and Hartmann, B. (2001) Quasi-Exactly Solvable $N \times N$-Matrix Schrödinger Operators. Modern Physics Letters A, 16, 1895-1906.

https://doi.org/10.1142/S0217732301005242 